Revue de droit comparé du travail et de la sécurité sociale

3 | 2019

Les migrations internationales de travail

\title{
La réforme du régime de l'assurance chômage : durcissement des conditions d'indemnisation et ouverture à de nouveaux bénéficiaires
}

\section{Clément Cailleteau}

\section{(2) OpenEdition}

Journals

Édition électronique

URL : https://journals.openedition.org/rdctss/1521

DOI : $10.4000 /$ rdctss. 1521

ISSN : 2262-9815

Éditeur

Centre de droit comparé du travail et de la sécurité sociale

Édition imprimée

Date de publication : 1 novembre 2019

Pagination : 200-205

ISSN : $2117-4350$

\section{Référence électronique}

Clément Cailleteau, «La réforme du régime de l'assurance chômage : durcissement des conditions d'indemnisation et ouverture à de nouveaux bénéficiaires », Revue de droit comparé du travail et de la sécurité sociale [En ligne], 3 | 2019, mis en ligne le 01 novembre 2021, consulté le 13 novembre 2021. URL : http://journals.openedition.org/rdctss/1521; DOI : https://doi.org/10.4000/rdctss.1521

\section{(c) (†) $\ominus$}

Revue de droit comparé du travail et de la sécurité sociale est mise à disposition selon les termes de la Licence Creative Commons Attribution - Pas d'Utilisation Commerciale - Pas de Modification 4.0 International. 


\section{CLÉMENT CAILLETEAU}

COMPTRASEC-UMR CNRS 5114, UNIVERSITÉ DE BORDEAUX

\section{LA RÉFORME DU RÉGIME DE L'ASSURANCE CHÔMAGE : DURCISSEMENT DES CONDITIONS D'INDEMNISATION ET OUVERTURE À DE NOUVEAUX BÉNÉFICIAIRES}

Le 18 juin 2019, la réforme du régime de l'assurance chômage a été présentée avec la haute ambition de "lutter contre la précarité " ${ }^{1}$. Au milieu de l'été, dans un flou relatif quant aux intentions du gouvernement, les deux décrets du 26 juillet $2019^{2}$ constituent ainsi l'aboutissement de ce processus de réforme ${ }^{3}$. Le premier procède à une abrogation de l'agrément de la convention d'assurance chômage du 14 avril 2017 dontil modifie largement les conditions d'indemnisation des travailleurs involontairement privés d'emploi. Il prévoit également « une modulation des contributions chômage patronales des entreprises pour celles relevant d'un secteur d'activité à taux de séparation très élevé ». Le second décret détaille notamment les conditions de la nouvelle allocation des travailleurs indépendants et les modalités de l'expérimentation du journal de la recherche d'emploi que le demandeur d'emploi devra renseigner lors du renouvellement mensuel de son inscription à la liste des demandeurs d'emploi.

Quelles sont les lignes directrices de cette réforme? Tout d'abord, les mesures engagées contre le recours aux contrats courts conduisent principalement au durcissement des conditions d'indemnisation des travailleurs précaires involontairement privés d'emploi (I). Ensuite, les mesures destinées à ouvrir l'assurance chômage à de nouveaux bénéficiaires restent assez timides et ne permettent pas de conclure à une transformation du modèle (II).

\section{I - UN DURCISSEMENT DES CONDITIONS D'INDEMNISATION DES TRAVAILLEURS PRÉCAIRES INVOLONTAIREMENT PRIVÉS D'EMPLOI}

Le premier objectif annoncé visait à lutter contre la fragmentation des contrats de travail. En effet, le gouvernement s'est appuyé sur le constat que les règles de l'assurance chômage pouvaient avoir des conséquences sur la structuration du "marché du travail ». Des dispositifs, tels le cumul emploi-chômage et le rechargement des droits, pouvaient

1 Et ce, malgré un lapsus. En effet lors de la conférence de presse du 18 juin 2019, la Ministre du travail, Muriel Pénicaud, a déclaré que la réforme était "contre le chômage et pour la précarité ", avant de se reprendre " contre la précarité ».

2 JORF nº 174 du 28 juillet 2019 - Décret n² 2019-796 du 26 juillet 2019 relatif aux nouveaux droits à indemnisation, à diverses mesures relatives aux travailleurs privés d'emploi et à l'expérimentation d'un journal de la recherche d'emploi et Décret n²019-797 du 26 juillet 2019 relatif au régime d'assurance chômage. Les dispositions entrent en vigueur à partir du $1^{\text {er }}$ novembre 2019.

3 Sur les débuts de ce processus de réforme, voir notamment M. Badel, «Indemnisation du chômage : la réforme est en marche ", Revue de droit comparé du travail et de la sécurité sociale, 2018/1, p. 60. 
conduire employeurs et salariés à préférer inscrire leurs relations dans le cadre d'une succession de contrats courts à temps plein, plutôt que choisir la solution de l'emploi continu à temps partiel.

Or cette approche, destinée à limiter l'impact des règles de l'indemnisation du chômage sur les pratiques d'emploi, a conduit au durcissement des conditions d'indemnisation. Trois mesures principales (au-delà de la dégressivité des allocations chômage pour les cadres) ${ }^{4}$ en sont la cause.

En premier lieu, pour bénéficier du droit à l'indemnisation, les personnes couvertes par l'assurance chômage devront avoir travaillé 6 mois (au moins 130 jours ou 910 heures) au cours des 24 derniers mois ${ }^{5}$ (auparavant 4 mois au cours des 28 derniers mois). Désormais, il faut donc avoir travaillé plus longtemps et sur une période de référence plus courte pour pouvoir prétendre à l'ouverture de droits à l'indemnisation d'une période de chômage.

À cette première réduction du périmètre des personnes protégées contre le risque social du chômage, le gouvernement a ajouté la réduction du cercle des personnes pouvant bénéficier du rechargement de leurs droits. Le seuil de déclenchement du rechargement des droits est relevé de 1 à 6 mois de travail nécessaire.

Enfin, la principale mesure est la modification du salaire de référence pour la détermination du montant de l'allocation journalière. Le salaire de référence pris en compte jusque-là était le salaire moyen des jours travaillés. Aujourd'hui, le salaire de référence sera le salaire moyen mensuel, quel que soit le nombre de jours travaillés ${ }^{6}$. En conséquence, les travailleurs en contrat à durée indéterminée seront peu concernés. La modification du salaire de référence pour le calcul de l'indemnité journalière aura un impact très fort sur le montant de l'allocation chômage servie aux travailleurs alternant des contrats courts et des périodes d'inactivité.

À ce stade, deux observations peuvent être formulées.

Tout d'abord, si l'effort du gouvernement consiste certes à réduire les contrats courts en considérant que les conditions d'indemnisation du chômage incitent à leur multiplication, il n'en demeure pas moins que les moyens employés n'épargnent pas le travailleur précaire involontairement privé d'emploi. La logique de la réforme conduit à modifier le salaire de référence en excluant les jours non travaillés, ce qui entraîne une diminution considérable du montant de l'allocation chômage. Sans parler de l'efficacité de cette mesure sur la réduction effective des contrats courts, il est permis de douter du véritable objectif des deux autres mesures visant à relever le seuil de déclenchement d'ouverture des droits

4 La réforme introduit également un coefficient de dégressivité dans le calcul de l'allocation d'aide au retour à l'emploi. Le montant diminuera à partir du $183^{\mathrm{e}}$ jour d'indemnisation avec un coefficient de dégressivité de 0,7 (art. 17 bis, Décret $n^{\circ}$ 2019-797). Toutefois, cette mesure de dégressivité ne concerne que les travailleurs qui gagnent plus de 4500 euros bruts en période d'activité.

5 Et au cours des 36 mois qui précèdent la fin du contrat de travail (terme du préavis) pour les salariés âgés de 53 ans et plus à la date de la fin de leur contrat de travail.

6 Sur l'exemple d'une personne travaillant 15 jours sur un mois et ne travaillant pas les 15 autres. Si elle gagne 740 euros, le salaire de référence pris en compte était de 1480 sur le mois avant la réforme. En conséquence, le montant de l'allocation chômage était de 920 euros. Avec la réforme, le salaire de référence pris en compte est 740 euros et en conséquence le montant de l'allocation sera de 460 euros. 
(durée d'affiliation et rechargement des droits). Celles-ci devraient conduire à une réduction inutile des bénéficiaires de l'allocation chômage, sans pour autant engendrer de véritables effets sur le recours aux contrats courts.

Bien sûr, l'orientation vers la limitation des contrats courts concerne aussi les employeurs avec la mesure de modulation des cotisations en fonction du taux de séparation?. Le taux de séparation compare le nombre d'inscriptions sur la liste des demandeurs d'emplois des travailleurs sortant de l'entreprise ${ }^{8}$ avec les effectifs de l'entreprise. Selon le taux de séparation calculé dans l'entreprise et en comparaison au taux de séparation moyen calculé dans le secteur d'activité, la cotisation patronale à l'assurance chômage sera majorée ou minorée - de 3 à 5,05 \% -. Il faut remarquer que si la réforme a des conséquences financières importantes pour un grand nombre de chômeurs, la modulation patronale ne concerne que certains secteurs d'activités 9 . II s'agit donc d'une réforme timide dans sa mise en œuvre qui s'ajoute à une «boîte à outils» (déjà très variée) de la lutte contre les contrats courts à répétition ${ }^{10}$.

Serait-ce une asymétrie dans l'entreprise réformatrice, selon que l'on vise salariés ou employeurs dans la responsabilité des pratiques de l'emploi? II n'est pas étonnant que I'on retrouve dans les réformes de l'assurance chômage une méfiance persistante à l'égard des travailleurs involontairement privés d'emploi. L'histoire de l'indemnisation du chômage rappelle qu'il s'agit d'un risque particulier puisque que, contrairement aux autres risques sociaux (pris en charge par la Sécurité Sociale) qui sont tous contingents au corps de l'assuré social ${ }^{11}$, le chômage est une situation. En ce sens, la durée de la réalisation de ce risque social dépend au moins pour partie du comportement de la victime. Les études portant sur l'histoire du chômage ont depuis longtemps montré que le chômage est une catégorie normative qui génère un contrôle important sur le caractère volontaire ou non de la privation d'emploi ${ }^{12}$. L'indemnisation du chômage est perçue comme une manifestation

7 Sur ce point, voir, C. Willmann, "Nouveau régime de cotisations d'assurance chômage: la modulation, une vraie fausse rupture ", Lexbase éd. sociale, $\mathrm{n}^{\circ}$ 793. Les modalités d'application de cette modulation sont définies aux articles 50-2 à 50-15 du règlement d'assurance chômage, annexé au décret 2019-797. La prise en compte de cette modulation concerne les entreprises de 11 salariés et plus.

8 Toutes les fins de contrat ne sont pas retenues, par ex. exclusion des démissions et des fins de contrat de mission. Voir. décret $n^{\circ}$ 2019-797, Annexe A, article 50-6.

9 Le gouvernement a retenu 7 secteurs.

10 Il existe déjà des mécanismes qui prennent en compte la nature et la durée du contrat pour fixer le taux de cotisation afférent au contrat. Le taux de la contribution à la charge des employeurs est fixé à 4,05\% (décret du 26 juillet 2019, Annexe A, art. 50-1, applicable au 1er novembre 2019). Par dérogation, la contribution à la charge de l'employeur est fixée à 4,55\% pour les contrats de travail à durée déterminée au titre des « contrats à durée déterminée d'usage » (article L. 1242-2 du Code du travail).

11 C'est un évènement d'atteinte au corps (maladie, vieillesse) qui entraîne la perte ou l'augmentation de la capacité de gains.

12 Voir C. Topalov, Naissance du chômeur, 1880-1910, éd. Albin Michel, 1994; R. Salais, N. Baverez et B. Reynaud, L'invention du chômage, PUF, 1986. 
de l'effet pervers ${ }^{13}$ des systèmes de protection sociale: c'est le niveau élevé de protection qui inciterait à rester en situation d'inactivité (sans que cela ne soit véritablement démontré). La réforme vise donc à durcir les voies de l'indemnisation de l'assuré et à sanctionner les employeurs à trop haut niveau de "séparation", pour s'assurer que les comportements des acteurs ne nuisent pas au système. On notera également l'expérimentation du journal de la recherche d'emploi pour les demandeurs d'emploi ${ }^{14}$. Cette mesure d'encadrement de la recherche d'emploi n'est qu'au stade de l'expérience mais on se demande quelles conséquences son maintien ou non pourrait entraîner à l'avenir pour l'actualisation des droits. En outre, cette mesure semble signer le renforcement du contrôle social à l'égard du comportement du demandeur d'emploi.

La seconde remarque porte sur l'efficacité même de la réforme. En termes économiques, les mesures de durcissement de l'indemnisation des travailleurs privés d'emploi répondent à un déséquilibre important dans les comptes de I'UNEDIC. Ce déséquilibre est lié aux contrats courts qui génèrent 2,664 milliards d'euros de contribution, mais entrainent 8,886 milliards d'euros de prestations ${ }^{15}$. II est clair que les mesures de durcissement de l'indemnisation des chômeurs semblent orientées vers un objectif économique de réduction de ces dépenses. Mais pour juger de l'efficacité sociale de la mesure, il faut se demander quelle partie de la population des travailleurs involontairement privés d'emploi est concernée. Hormis les chômeurs de catégories A (personnes sans emploi et tenues d'en chercher), soit environ 3,4 millions de personnes dont les demandeurs d'emploi de longue durée ${ }^{16}$, tous les autres chômeurs sont dans des situations où ils alternent périodes d'activité et d'inactivité. Cela concerne près de 2,2 millions de personnes et des situations professionnelles très hétérogènes (selon l'âge, le parcours professionnel).

L'analyse du caractère involontaire de la privation d'emploi en est d'autant plus compliquée. Ces personnes ont-elles vraiment le choix de leurs conditions d'emploi ou subissent-elles leurs situations dites de précarité? La réponse du gouvernement se trouve dans les décrets: cette population n'est pas irréductible à l'emploi stable et régulier et, tel un phénomène de Cana, les réformes prétendent parvenir à clarifier définitivement les situations faites de mix confus emploi-chômage en emploi stable. Pour d'autres, au contraire, cette population importante qui alterne chômage et activité n'est pas réductible à l'intransigeant dilemme catégoriel: emploi ou chômage ${ }^{17}$. La fragilisation de l'accès à l'indemnisation du chômage et l'abaissement du niveau d'indemnisation apparaissent

13 Voir A. O. Hirschman, Deux siècles de rhétorique réactionnaire, éd. Fayard, 1991, p. 28: "L'effet pervers, ce qu'il entend démontrer, c'est que les mesures destinées à faire avancer le corps social dans une certaine direction, le feront effectivement bouger, mais dans le sens inverse ».

14 Décret $n^{\circ}$ 2019-796 du 26 juillet 2019 relatif aux nouveaux droits à indemnisation, à diverses mesures relatives aux travailleurs privés d'emploi et à l'expérimentation d'un journal de la recherche d'emploi, art. 7

15 V. Unédic, "Comprendre l'assurance chômage en 9 fiches », septembre 2017, fiche 6, p. 3, cité par J-Y. Kerbourc'h, "Indemnisation du chômage et déclin de la logique assurantielle », Droit social, juillet-août 2018, numéro spécial : " assurance chômage: un nouveau modèle? », p. 607.

16 Source: Pôle emploi-Dares, STMT, données CVS-CJO, 2 e trimestre 2019.

17 Voir notamment une tribune de F-X. Petit, directeur général de Matrice, «Emploi, chômage: nous vivons un effondrement de notre manière de formuler le social », Le Monde, 14 juillet 2019: «En fait, nous vivons un effondrement de notre manière de formuler le social. Emploi contre chômage ne décrivent plus la réalité (...). Le mix emploi/non-emploi est la réalité des millions d'actifs ». 
comme une mise en danger d'une large partie des chômeurs. La lutte contre la multiplication des contrats courts peut passer par d'autres moyens que l'affaiblissement de la couverture du régime d'assurance chômage ${ }^{18}$. II serait sans doute préférable d'opter pour la voie de la régulation dans le cadre de la négociation collective.

\section{II - UNE TIMIDE OUVERTURE DE L'ASSURANCE CHÔMAGE À DE NOUVEAUX BÉNÉFICIAIRES}

La réforme vise aussi à ouvrir le régime d'assurance chômage à de nouveaux bénéficiaires, notamment dans certains cas de démission. En application de la loi « pour la liberté de choisir son avenir professionnel » du 5 septembre 2018, le décret $n^{\circ}$ 2019-796 du 26 juillet 2019 prévoit les modalités de l'accès à l'indemnisation du chômage pour les démissionnaires en reconversion professionnelle. Le décret précise notamment les critères d'attribution du caractère réel et sérieux d'un projet professionnel par les commissions paritaires interprofessionnelles régionales ${ }^{19}$.

En outre, il s'agit aussi de l'ouverture aux indépendants. Le décret n²019-796 du 26 juillet 2019 fixe les conditions d'accès à la nouvelle «allocation des travailleurs indépendants ». Le fait générateur de l'ouverture des droits est la date de cessation d'une activité non-salariée. Plusieurs autres conditions y sont assorties. Le travailleur indépendant doit avoir exercé cette activité durant une période minimale et ininterrompue de deux ans au titre d'une seule et même entreprise. Il doit être effectivement à la recherche d'un emploi. Il doit justifier, au titre de l'activité non-salariée, de revenus antérieurs dractivité égaux ou supérieurs à 10000 euros par an. Il doit également justifier dıautres ressources inférieures au montant forfaitaire mensuel mentionné à l'article L. 262-2 du Code de l'action sociale et des familles, applicable à un foyer composé dıune personne seule.

Ces ouvertures à de nouveaux bénéficiaires restent limitées à des conditions d'accès très particulières. II ne s'agit en aucun cas de la concrétisation d'une " assurance chômage universelle », à laquelle le Président de la République s'était engagée durant sa campagne, mais davantage d'une extension limitée ${ }^{20}$ qui ne change pas fondamentalement l'assurance chômage en un nouveau droit de la personne ouvert à tous les actifs.

En définitive, le changement de modèle annoncé apparaît comme un mirage. Certes, il y a bien une ouverture à de nouveaux bénéficiaires aux côtés du travailleur involontairement privé d'emploi. De manière limitée, l'assurance chômage bénéficie désormais à des indépendants et à des travailleurs salariés qui décident de se priver volontairement d'emploi pour un projet de reconversion professionnelle.

18 Voir D. Baugard, B. Coquet et É. Heyer, « Quels instruments juridiques pour limiter le recours aux contrats courts?», RDT, 2019, p. 452.

19 Voir Décret $n^{\circ} 2019-796$ du 26 juillet 2019, art. 1.

20 Voir C. Cadoret, L. Caussat et E. Robert, « L'assurance chômage est-elle en voie d'universalisation? », RFAS, 2018/4, p. 223. 


\section{FRANCE}

Mais dans la réalité, cette réforme vise sans doute à diminuer davantage l'accès et l'étendue de la couverture contre le risque chômage. Alors qu'elle prétend lutter contre le recours aux contrats courts, elle durcit les conditions d'indemnisation des travailleurs précaires involontairement privés d'emploi. Il s'agit, encore une fois, d'un retour à un élément moral dans le diagnostic des réformes de l'assurance chômage. Portées par l'argument de «l'effet pervers» (en considérant que les anciennes conditions d'indemnisation étaient trop incitatives à rester volontairement au chômage), il reste à espérer que les nouvelles conditions d'indemnisation ne conduisent pas à « mettre en péril $»^{21}$ un nombre important de travailleurs précaires involontairement privés d'emploi.

21 Voir A. O. Hirschman, Deux siècles de rhétorique réactionnaire, Fayard, 1991. 